\title{
The Differential Effects of Reward on Space- and Object-Based Attentional Allocation
}

\author{
Jeongmi Lee and Sarah Shomstein \\ Department of Psychology, George Washington University, Washington, DC 20052
}

Estimating reward contingencies and allocating attentional resources to a subset of relevant information are the most important contributors to increasing adaptability of an organism. Although recent evidence suggests that reward- and attention-based guidance recruits overlapping cortical regions and has similar effects on sensory responses, the exact nature of the relationship between the two remains elusive. Here, using event-related fMRI on human participants, we contrasted the effects of reward on space- and object-based selection in the same experimental setting. Reward was either distributed randomly or biased a particular object. Behavioral and neuroimaging results show that space- and object-based attention is influenced by reward differentially. Space-based attentional allocation is mandatory, integrating reward information over time, whereas object-based attentional allocation is a default setting that is completely replaced by the reward signal. Nonadditivity of the effects of reward and object-based attention was observed consistently at multiple levels of analysis in early visual areas as well as in control regions. These results provide strong evidence that space- and object-based allocation are two independent attentional mechanisms, and suggest that reward serves to constrain attentional selection.

\section{Introduction}

Recent investigations demonstrate that the neural correlates of reward (Schultz et al., 1998; Hikosaka, 2007) and attentional selection (Kastner and Ungerleider, 2000; Corbetta and Shulman, 2002; Serences et al., 2005) are both associated with a network of areas engaging parietal and frontal cortices, midbrain, and striatum. Furthermore, the consequences of reward-based (Shuler and Bear, 2006; Serences, 2008) and attention-based selection (Ress et al., 2000; Müller and Kleinschmidt, 2003; Shomstein and Behrmann, 2006) are similar, both modulating neural activity in corresponding sensory regions. Although it is well established that voluntary behaviors are strongly modulated by reward, it remains unclear whether similar reward-based modulation occurs for perceptual processes, such as attention, that are involuntary and automatic (Della Libera and Chelazzi, 2006; Hickey et al., 2010). The important unanswered question is whether attentional allocation occurs mandatorily, independent of reward manipulations, or whether it is modulated by reward. Understanding whether reward provides an informative signal that constrains or even overrides involuntary attentional allocation will elucidate the mechanism by which multiple sources are integrated for the purpose of selection.

Here, we focus exclusively on two fundamental mechanisms of attentional allocation (Posner, 1980; Egeth and Yantis, 1997),

Received Dec. 4, 2012; revised April 15, 2013; accepted May 9, 2013.

Author contributions: J.L. and S.S. designed research; J.L. and S.S. performed research; J.L. and S.S. analyzed data; J.L. and S.S. wrote the paper.

This work was supported by National Science Foundation Grant BCS-1059523 and National Institutes of Health Grant R21-EY021644 to S.S. We thank Marlene Behrmann, George Malcolm, and Fatma Uyar for comments and suggestions.

The authors declare no competing financial interests.

Correspondence should be addressed to Dr. Jeongmi Lee, Department of Psychology, George Washington University, 2125 G Street NW, Washington, DC 20052. E-mail: jeongmi@gwmail.gwu.edu.

DOI:10.1523/JNEUROSCI.5575-12.2013

Copyright $\odot 2013$ the authors $\quad 0270-6474 / 13 / 3310625-09 \$ 15.00 / 0$ space- and object-based, and present data from a series of eventrelated functional magnetic resonance imaging (fMRI) experiments in which the effects of reward on space- and object-based selection are investigated. To this end, a variant of the wellestablished two-rectangle paradigm (Egly et al., 1994; Moore et al., 1998) was adopted, which has consistently demonstrated the presence of two automatic effects of attentional allocation: (1) space-based effect, indicating that the distance between the cued location and the target affects perceptual efficiency (Müller and Kleinschmidt, 2003; Shomstein and Behrmann, 2006); and (2) object-based effect, indicating that, when part of an object is attended, the rest of the object benefits perceptually (Behrmann et al., 1998; Moore et al., 1998; Shomstein and Yantis, 2004).

The two-rectangle paradigm was juxtaposed with three different monetary reward schedules. Because identical visual stimuli (with identical probabilities of occurrence) were presented in all reward schedules, any observed differences in behavioral or neural activity can only be attributed to the influence of reward on attentional orienting. We directly examined changes observed over retinotopically organized visual cortex (V1-V4) and reward- and attention-related regions identified in the whole-brain analysis as a function of target type and reward schedule. Sensory responses to the cue and to the target were separately analyzed to examine whether changes observed are target-specific or reflect cue-related preparatory processes that persist until target identification.

We hypothesize that, if reward provides an informative signal that constrains attentional allocation, it will influence attentional guidance in one of two ways: (1) reward signal will modulate the magnitude of attentional allocation; or (2) it will replace the attentional signal entirely. Importantly, the two separate scenarios will also shed light on whether automatic space- and object-based attentional selection is mandatory, or is a mere default (space- or object-based attention is abandoned in favor of reward). 


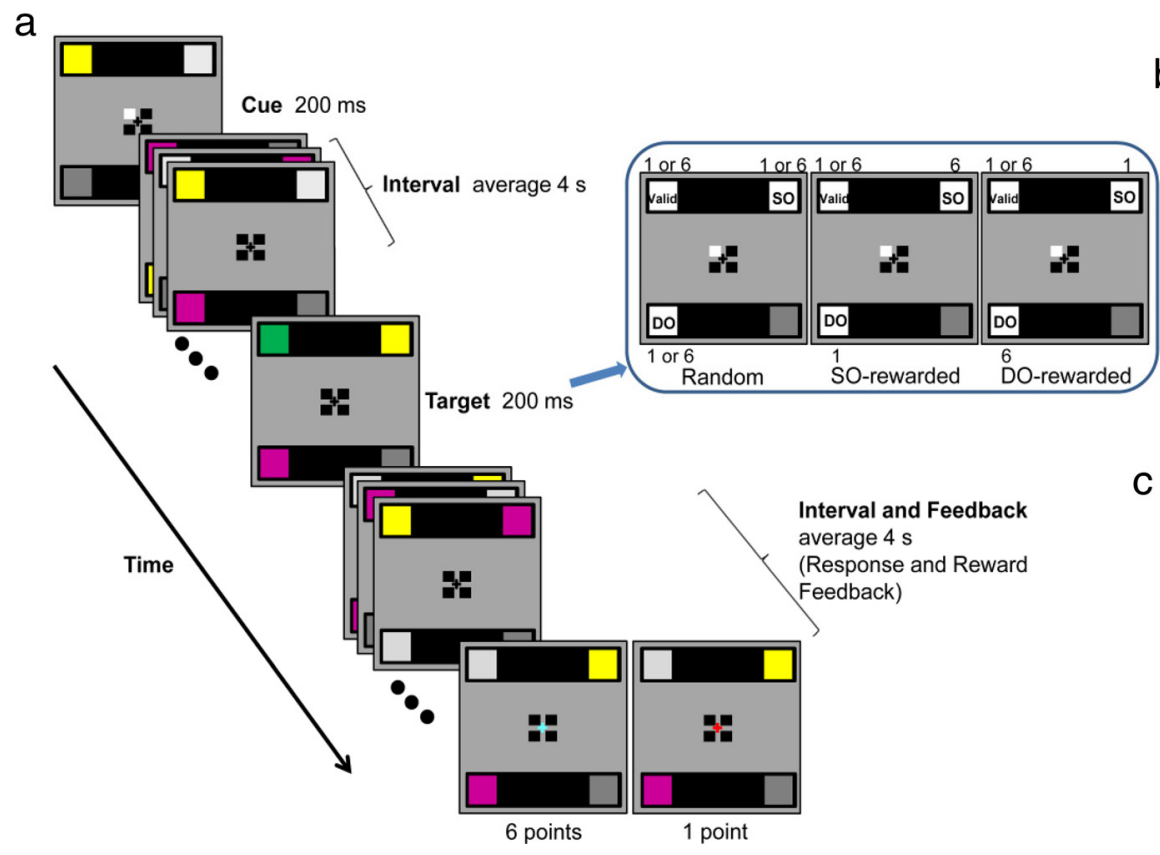

b

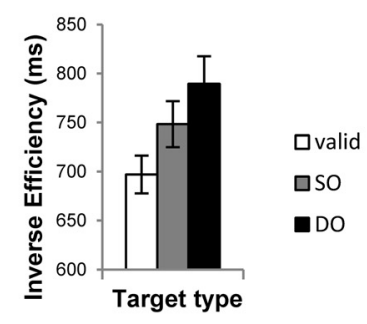

Figure 1. $a$, Experimental design. Participants maintained fixation on the center cross. The color of one of the four black cue squares changed to white for $200 \mathrm{~ms}$, indicating that target color (blue or green) will appear at the corresponding color patch. After the cue, during an average of $4 \mathrm{~s} \mathrm{jittered} \mathrm{interval,} \mathrm{the} \mathrm{color} \mathrm{within} \mathrm{each} \mathrm{color} \mathrm{patch} \mathrm{changed} \mathrm{synchronously} \mathrm{every} 200 \mathrm{~ms}$. After the interval, target color appeared for $200 \mathrm{~ms}$ at one of three possible locations (valid, S0, or D0) with 60\%, 20\%, and 20\% chance, respectively. During another jittered interval (average $4 \mathrm{~s}$ ), participants responded to target. After a response, reward amount was indicated by the color of the fixation cross: cyan represents 6 points; red, 1 point; black, incorrect responses ( 0 point). There were three monetary reward schedules: (1) random reward, where $\mathrm{SO}$ and DO targets are rewarded with random distribution of high (6 points) and low (1 point) rewards; (2) S0-rewarded schedule, where SO targets receive high reward ( 6 points) while DO targets receive low reward (1 point); and (3) DO-rewarded schedule, where D0 targets receive high reward (6 points) while S0 targets receive low reward (1 point). Valid targets receive random distribution of high (6 points) and low (1 point) rewards in all three reward schedules. $\boldsymbol{b}$, Inverse efficiency for each target type (valid, S0, D0), obtained from a behavioral experiment without reward outside of the scanner. Here, and in all figures, error bars represent SEM. c, Inverse efficiency for each target type (valid, S0, D0) in each reward schedule (Random, S0-rewarded, DO-rewarded), obtained from the fMRl experiment.

\section{Materials and Methods}

Subjects. Sixteen (9 females, 19-22 years of age) and 14 (11 females, 21-31 years of age) human adults, with normal or corrected-to-normal vision, and no history of neurological problems, participated in the behavioral study and fMRI study, respectively. All participants gave informed consent, and the study was approved by the Institutional Review Board of the George Washington and Georgetown Universities. Each participant in the fMRI study was trained outside the scanner and then participated in two separate $1.5 \mathrm{~h}$ fMRI sessions. Data from two participants were discarded because of poor behavioral performance and excessive head motion in the scanner.

Experimental task. Stimulus displays (Fig. 1a) consisted of two black rectangles $\left(8.49^{\circ} \times 1.34^{\circ}\right)$ oriented either horizontally or vertically (counterbalanced among participants) on a gray background and centered $3.58^{\circ}$ to the left and right, or above and below a black fixation cross $\left(0.57^{\circ} \times 0.57^{\circ}\right)$. Each end of the rectangles contained a color patch $\left(1.24^{\circ} \times\right.$ $\left.1.24^{\circ}\right)$ on which target and distractor colors appeared, centered $4.99^{\circ}$ diagonally to a fixation cross. Four black cue squares $\left(0.48^{\circ} \times 0.48^{\circ}\right)$ were centered $0.48^{\circ}$ diagonally to a fixation cross with a distance of $4.51^{\circ}$ to the corresponding color patch.

Each trial began with a cue with one of the cue squares changing its color to white for $200 \mathrm{~ms}$, indicating with $60 \%$ certainty that a target will appear at the corresponding color patch. After a cue, during a pseudorandomly jittered ( $3-5 \mathrm{~s}$ in $200 \mathrm{~ms}$ intervals, average $4 \mathrm{~s}$ ) interval, the color patch in each of the four locations changed its color synchronously every $200 \mathrm{~ms}$. Distractor colors were chosen randomly among gray, silver, yellow, and purple with the restrictions that no identical color was present in two locations simultaneously and that there were no immediate repeats of the same color at the same location. After the interval, green or blue target color appeared for $200 \mathrm{~ms}$ in one of the three possible locations, the location indicated by the cue (valid), in the same object as the valid location but at a different end (SO), or in the different object at the location equidistant from the valid location (DO). The target color appeared in the valid location on $60 \%$, in the SO on $20 \%$, and in the DO on $20 \%$ of the trials. After the target, and during pseudorandomly jittered interval ( $3-5 \mathrm{~s}$ in $200 \mathrm{~ms}$ intervals, average $4 \mathrm{~s}$ ) in which the colors on color patches kept changing every $200 \mathrm{~ms}$ with the same restrictions, participants responded as quickly and accurately as possible by pressing the left button for a green target and the right button for a blue target. Participants held two button boxes, one in each hand. After a response, participants received feedback indicating the reward amount signified by the change of color of the fixation cross: cyan for 6 points earned, red for 1 point earned, and no change in color for incorrect or missing responses ( 0 point).

Three different reward schedules (Fig. 1a) were used for each participant: random, SO-rewarded, and DO-rewarded: random, 1 or 6 points were randomly awarded to correctly identified SO and DO targets; SOrewarded, SO targets received a reward of 6 points, and DO targets were rewarded with 1 point; DO-rewarded, DO targets were rewarded with 6 points, and SO targets were rewarded with 1 point. Valid targets were rewarded with 1 or 6 points randomly (to ensure validity effects) in all three reward schedules. In two separate $1.5 \mathrm{~h}$ fMRI sessions, random reward schedule was always performed on session 1 to prevent influences from biased reward schedules, and SO-rewarded and DO-rewarded schedules were performed on session 2 in a counterbalanced order. Participants were explicitly informed of the biases of each reward schedule and performed 4 runs for each reward schedule.

During a break after each run, the cumulative number of points earned was presented on the screen to encourage performance. Participants were told that they would be paid $\$ 70$ for a basic payment, with an additional incentive between $\$ 0$ and $\$ 30$ based on cumulative points earned. In fact, all participants were paid $\$ 100$ upon completion of the two $1.5 \mathrm{~h}$ sessions and were fully debriefed at the end of the experiment.

fMRI acquisition and analysis. MRI scanning was performed on a Siemens 3-Tesla scanner equipped with a 12-channel head coil at the Center for Functional and Molecular Imaging (CFMI) at the Georgetown University Medical Center. Each scan lasted $441 \mathrm{~s}$, consisting of $10 \mathrm{~s}$ fixation 
followed by 50 experimental trials. Data from the experiment were collected in 12 scans per participant. Whole-brain echoplanar functional images were acquired in 28 transverse slices $(\mathrm{TR}=1500 \mathrm{~ms}, \mathrm{TE}=30 \mathrm{~ms}$, matrix $=64 \times 64$, field of view $=200 \times 200 \mathrm{~mm}^{2}$, slice thickness $=4$ $\mathrm{mm}, 0 \mathrm{~mm}$ gap $)$. High-resolution anatomical images $(1 \times 1 \times 1 \mathrm{~mm}$ resolution) were acquired by using MPRAGE T1-weighted sequence $\left(\mathrm{TR}=2530 \mathrm{~ms}, \mathrm{TE}=3.5 \mathrm{~ms}\right.$, field of view $=256 \times 256 \mathrm{~mm}^{2}$, matrix $=$ $256 \times 256$, number of slices $=176)$.

Data analysis was performed using BrainVoyager QX (version 2.3.0, Brain Innovation). Images from each functional run were slice time corrected, motion corrected, and then filtered [Gaussian FWHM 3 s, Highpass (general linear model-Fourier) 2 sines/cosines] to remove lowfrequency and high-frequency noise in the functional time series. All echoplanar functional images and anatomical images were transformed into the standardized Talairach and Tournoux (1988) atlas space. The cortical surface of each participant was corrected for inhomogeneity, reconstructed by segmenting the right and left hemispheres, segmenting white from gray matter, and inflating the cortical sheet. Separate retinotopic mapping scan was performed for each participant by using established procedures (Sereno et al., 1995) to define borders between early visual areas (V1-V4) on the inflated brain. Then, patches of interest (POIs) were drawn within the four visual areas by a separate localizer scan in which flickering checkerboards $(4 \mathrm{~Hz})$ were presented at the exact locations as the four color patches in the experiment.

Cue- and target-related activity was deconvolved to separately examine preparatory effects of attention following the cue and sensory changes specific to target processing and identification. For target-related analysis, neural activity elicited within the POIs at the time of the target onset was evaluated for each target type. For cue-related analysis, we examined neural activity elicited within the POIs corresponding to spatial locations of the upcoming target immediately after the cue onset. For example, for horizontally oriented rectangles and the top left cue, BOLD responses from top-left, top-right, and bottom-left POIs were extracted as valid, $\mathrm{SO}$, and DO values, respectively. For both target- and cue-related activity, $\beta$-coefficients were extracted separately from each POI (top-left, topright, bottom-left, bottom-right) in each visual area (V1-V4), by using a general linear model and a design matrix that modeled the response to the target or the cue event. The blood oxygenation level-dependent (BOLD) response was estimated at the time of target or cue onset and at each of the next 11 time points, $0-16.5 \mathrm{~s}$ after stimulus, averaged for each target event in each reward schedule, and plotted against time. Then, within each visual area, responses for each condition were collapsed over the four POIs. Peak response was calculated by averaging $\beta$-coefficients at time points $6-7$ for target-related activity and by averaging $\beta$-coefficients at time points 6-9 for cue-related activity. Different time points were used for target- and cue-related activity based on the shape of the mean BOLD time courses that showed the peak for cue-related activity appearing relatively later than that for target-related activity. This delay was expected given the use of endogenous centrally located cues and targets appearing peripherally. Because cue-related activity was extracted at the time of the cue onset from POIs corresponding to locations of the upcoming target that are spatially different from the location of the cue, the preparatory effects of selective attention following the cue were expected to be slightly delayed.

For multivoxel correlation analysis, data were exported to MATLAB (MathWorks) using BrainVoyager's BVQXtools MATLAB toolbox, and all subsequent analyses were done in MATLAB following the method of Haxby et al. (2001). First, the POIs across visual cortex (V1-V4) were combined into a single POI, creating four combined POIs (top left, top right, bottom left, bottom right). Then, separately for each subject, reward schedule, and combined POI, data were split into odd and even runs dataset. For each dataset separately, the mean response across all target types was subtracted from the responses to individual target types, normalizing each voxel's response. Next, the voxelwise response patterns for each of the target types in the odd runs were correlated with each of the target types in the even runs, generating a matrix of nine correlation coefficients. Finally, the correlation matrix was averaged across four POIs (top left, top right, bottom left, bottom right), yielding one averaged correlation matrix per each reward schedule.
Table 1. Regions activated for high reward versus low reward targets

\begin{tabular}{lrrrccc}
\hline Anatomical region & \multicolumn{1}{c}{$x$} & \multicolumn{1}{c}{$y$} & \multicolumn{1}{c}{$z$} & $t$ & $p$ & Brodmann's area \\
\hline rFEF & 35 & 8 & 48 & 3.88 & $<0.005$ & 6 \\
rInsula & 34 & 15 & 6 & 5.76 & $<0.001$ & 13 \\
rMFG & 14 & 46 & 12 & 5.21 & $<0.001$ & 10 \\
rACC & 11 & 28 & 24 & 5.94 & $<0.001$ & 32 \\
rSFG & 2 & 13 & 48 & 5.90 & $<0.001$ & 6 \\
Left precuneus & -19 & -47 & 39 & 7.68 & $<0.001$ & 31 \\
Left middle occipital gyrus & -31 & -86 & 12 & 5.57 & $<0.001$ & 19 \\
IIPL & -43 & -56 & 39 & 4.88 & $<0.001$ & 40 \\
\hline
\end{tabular}

Coordinates $(x, y$, and $z$ ) are reported in Talairach space (Talairach and Tournoux, 1988).

A whole-brain, random-effects group analysis was performed after applying a $4 \mathrm{~mm}$ FWHM spatial smoothing kernel to the functional data from each participant. Reward-sensitive regions were derived by contrasting high reward targets (SO target in SO-rewarded schedule and DO target in DO-rewarded schedule) and low reward targets (DO target in SO-rewarded schedule and SO target in DO-rewarded schedule). Statistical maps were computed by comparing the mean fit coefficients across $4.5-12 \mathrm{~s}$ after stimulus for each condition. The single-voxel threshold was set to $t_{(11)}=3.06$ and $p<0.01$. A minimum cluster size of 10 contiguous voxels $\left(257 \mathrm{~mm}^{3}\right)$ was adopted to correct for multiple comparisons, yielding a mapwise false-positive probability of $p<0.05$. This estimate was reached by performing 1000 Monte Carlo simulations of wholebrain statistical maps, using Brainvoyager's cluster threshold estimator (Goebel et al., 2006). The peak positions of the activations are reported in Table 1, and all coordinates correspond to the atlas of Talairach and Tournoux (1988).

Statistical analysis. Significance thresholds were set at $p<0.05$. Preliminary analysis revealed no significant main effect or interactions involving rectangle orientation $(F<1)$, collapsing data across this factor. Accuracy analysis revealed no significant main effects or interactions, reflecting the high degree of training participants had undergone before scanning. For behavioral analysis, a repeated-measures ANOVA was performed with reward schedule (random, SO-rewarded, DO-rewarded) and target type (valid, SO, DO) as within-subject factors and inverse efficiencies (IEs) as the dependent measure. For target- and cue-related activity analyses, repeated-measures ANOVAs were conducted, with visual area (V1-V4), reward schedule (random, SO-rewarded, DOrewarded), and target type (valid, SO, DO) as within-subject factors and peak response as the dependent measure. Planned comparisons followed according to the results of the ANOVAs.

Space-based effects were assessed by comparing responses to valid and invalid (SO and DO targets combined) targets. Object-based effects were assessed by comparing responses to $\mathrm{SO}$ and $\mathrm{DO}$ targets. The additivity of the modulation effects of reward and attentional allocation was examined by a repeated-measures ANOVA with reward schedule (SO-rewarded, DOrewarded) as a within-subject factor and the magnitude of modulation effect (mean response for low-rewarded target subtracted from mean response for high-rewarded target) as a dependent measure.

\section{Results}

\section{Behavior}

Two automatic effects of attentional allocation have been consistently demonstrated with a standard two-rectangle paradigm: (1) space-based effect, with valid targets identified faster and eliciting greater neural activity than invalid targets (Behrmann et al., 1998; Müller and Kleinschmidt, 2003; Shomstein and Behrmann, 2006); and (2) object-based effect, evidenced by faster detection and greater neural activity for SO targets than for DO targets, even though both are equidistant from the cued location (Behrmann et al., 1998; Moore et al., 1998; Shomstein and Yantis, 2004). We first conducted a behavioral experiment without reward, outside of the scanner, to validate the adopted paradigm as an effective space- and object-based attention task (Fig. 1b). To control for possible speed-accuracy trade-offs, reaction time 
(RT) and accuracy were combined into a single parameter of IE (Townsend and Ashby, 1983), calculated as the mean correct RT divided by accuracy rate, separately for each participant and each condition (Kiss et al., 2009; Kristjansson et al., 2010). ANOVA revealed a significant difference in mean IEs between target types $\left(F_{(2,30)}=6.45, p<0.02\right)$. The presence of a space-based effect was confirmed by faster mean IEs for valid targets $(697 \mathrm{~ms})$ compared with invalid targets $(769 \mathrm{~ms})\left(F_{(1,15)}=6.59, p<0.03\right)$. A significant object-based effect was also confirmed with SO targets (748 $\mathrm{ms})$ identified faster than DO targets $(790 \mathrm{~ms})\left(F_{(1,15)}=5.90, p<\right.$ $0.03)$. RT results showed the exact same pattern as IE results; a spacebased effect was confirmed by faster mean RTs for valid targets (659 $\mathrm{ms})$ compared with invalid targets $(708 \mathrm{~ms})\left(F_{(1,15)}=16.56, p<\right.$ 0.01 ), and a significant object-based effect was also observed with $S O$ targets $(695 \mathrm{~ms})$ identified faster than DO targets $(720 \mathrm{~ms})\left(F_{(1,15)}=\right.$ $10.17, p<0.01)$. The overall accuracy rate was $94 \%$ with no significant difference between target types.

Having validated the experimental paradigm as capable of eliciting robust space- and object-based effects, participants performed the task while being scanned with three reward schedules (Fig. 1a). We hypothesized that, if attentional allocation is a mere default setting that can be overridden in the presence of a more efficient strategy, space- and object-based selection will be abandoned in lieu of reward, such that modulatory effects will be exclusively guided by the reward manipulation. Thus, spacebased effects would contribute to attentional allocation only in random reward schedule (where reward does not provide additional information) and not exert its influence in SO- and DOrewarded schedules (where reward structure provides strategic advantage to abandon it). For object-based effects, it will disappear in random reward schedule (as both SO and DO locations are rewarded equally), whereas in SO- and DO-rewarded schedules, highly rewarded targets will benefit regardless of whether they appear in the already attended object. More importantly, there will be no interaction involving the magnitude of modulation effect between SO- and DO-rewarded schedules, as the difference between SO and DO targets is completely driven by reward structure in both reward schedules.

Alternatively, if attentional allocation occurs mandatorily, then the modulatory effects of reward and attentional guidance would manifest in an additive manner. Thus, the magnitude of space-based effects will be the same across different reward schedules because the pay-off structures for valid versus invalid targets are the same in all three reward schedules. On the other hand, the magnitude of objectbased effects (difference between SO and DO targets) will be different based on the reward schedules, leading to a significant interaction involving the magnitude of modulation effect between the SO- and DO-rewarded schedules. Specifically, in random reward schedule, we should observe an object-based effect of a typical magnitude (mandatory object-based effect). In SO-rewarded schedule, the magnitude of modulation will be exaggerated because of the additive effect of the reward schedule favoring SO targets. In DOrewarded schedule, given the conflict with reward favoring DO targets while attention favors SO targets, the magnitude of the modulation effect will be reduced or eliminated. Thus, significant interaction involving the magnitude of modulation effect will be observed in the additivity analysis.

ANOVA of behavioral data collected from within the scanner during the fMRI experiments (Fig. 1c) revealed no significant main effects but a significant reward schedule by target type interaction $\left(F_{(4,44)}=7.33, p<0.01\right)$. Planned comparisons were conducted for each reward schedule. A space-based effect was significant only in random reward schedule, evidenced by faster
IEs for valid targets (608 ms) than for invalid targets (647 ms) $\left(F_{(1,11)}=13.53, p<0.01\right)$, whereas space-based effects were absent in SO- and DO-rewarded schedules. These results suggest that space-based allocation is a default setting as it occurs when reward does not provide additional information (random reward) but disappears when reward structure provides strategic advantage to abandon it (SO- and DO-rewarded schedule).

For object-based effects, in random reward schedule, IEs for SO (646 ms) and DO (648 ms) targets were not significantly different, showing the absence of traditional object-based effect when all target locations are rewarded randomly and equally. The absence of object-based effect in random reward schedule provides the strongest support for the conclusion that object-based effects do not occur mandatorily, as it demonstrates that, in the presence of an alternative reward-based structure (even if reward biases two objects to the same extent), object-based representations fail to contribute to attentional guidance. In SO-rewarded schedule, SO targets $(656 \mathrm{~ms})$ were identified faster than DO targets $(714 \mathrm{~ms})\left(F_{(1,11)}=9.54, p<0.02\right)$, consistent with the direction of the traditional object-based effect. In DO-rewarded schedule, IEs for DO targets $(648 \mathrm{~ms})$ were faster than those for SO targets $(700 \mathrm{~ms})\left(F_{(1,11)}=5.63, p<0.04\right)$, indicating that the reward schedule biased toward the different-object location reversed the direction of the traditional object-based effect, with DO targets being detected most efficiently. RT results showed the exact same pattern as IE results (the same pattern of statistical significance), with the following mean values for valid, SO, and DO targets, respectively: random reward, $578 \mathrm{~ms}, 607 \mathrm{~ms}, 617 \mathrm{~ms}$; SO-rewarded, $648 \mathrm{~ms}, 634$ ms, $670 \mathrm{~ms}$; and DO-rewarded, $628 \mathrm{~ms}, 660 \mathrm{~ms}, 624 \mathrm{~ms}$. The overall accuracy rate was $94.2 \%$ with no significant difference between reward schedules and target types.

To assess additivity of the modulatory effects of reward and object-based attention, ANOVA was conducted with reward schedule (SO-rewarded, DO-rewarded) as a within-subject factor and the magnitude of modulation effect (mean IEs for lowrewarded target subtracted from mean IEs for high-rewarded target) as a dependent measure. The results indicated that the magnitude of modulation effect was not significantly different between SO- and DO-rewarded schedules, providing evidence that reward and object-based attention did not exert influence in an additive manner. Instead, it strongly suggests that objectbased attentional allocation is a default setting as it is abandoned in the presence of an alternative reward-based strategy, such that reward schedule exclusively modulates behavioral performance.

\section{Neuroimaging results}

\section{Target-related activity}

To examine the effects of reward and automatic attentional guidance tied to target processing, neural activity elicited within POIs at the time of the target onset was evaluated. BOLD time courses for each target type in each reward schedule were averaged over all subjects for each visual area. Peak response to target was calculated and submitted to ANOVA (Fig. 2). There was a significant reward schedule $\times$ target type interaction $\left(F_{(4,44)}=8.04\right.$, $p<0.05)$ and a three-way interaction between visual area, reward schedule, and target type $\left(F_{(12,132)}=1.94, p<0.04\right)$, indicating that the pattern of neural responses to each target type varied with reward schedule and visual area.

Planned comparisons were further conducted for each visual area and reward schedule. Consistent with the behavioral results, a space-based effect was significant only in random reward schedule, evidenced by greater responses for valid targets than for invalid targets $\left(\mathrm{V} 1, F_{(1,11)}=11.22, p<0.01 ; \mathrm{V} 2, F_{(1,11)}=7.31, p<\right.$ 


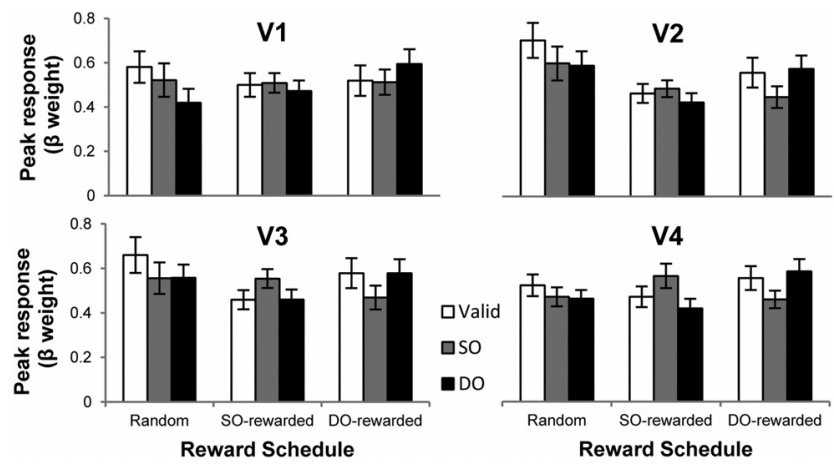

Figure 2. Target-related peak responses to each target type (valid, SO, D0) in each reward schedule (Random, S0-rewarded, D0-rewarded) in each visual area (V1-V4).

$\left.0.03 ; \mathrm{V} 3, F_{(1,11)}=4.19, p=0.065\right)$, whereas space-based effects were absent in SO- and DO-rewarded schedules in all visual areas. These results suggest that a space-based effect is a default setting that occurs when reward does not provide information preferentially biasing a specific location (random reward) but disappears when reward structure provides strategic advantage to abandon it (SO- and DO-rewarded schedule).

For object-based effects, planned comparisons revealed that, in $\mathrm{V} 2$, V3, and V4, the responses to SO and DO targets showed different patterns depending on the reward schedule: random, no significant difference between responses to $\mathrm{SO}$ and $\mathrm{DO}$ targets in V2, V3, and $\mathrm{V} 4$ (all $F<1$ ); SO-rewarded, greater response for SO than DO targets in $\mathrm{V} 2\left(F_{(1,11)}=7.40, p<0.02\right)$, V3 $\left(F_{(1,11)}=19.75, p<0.001\right)$, and $\mathrm{V} 4\left(F_{(1,11)}=8.56, p<0.02\right)$, consistent with the direction of the traditional object-based effect; and DO-rewarded, greater response for DO than SO targets in V2 $\left(F_{(1,11)}=11.69, p<0.01\right), \mathrm{V} 3\left(F_{(1,11)}=\right.$ $10.62, p<0.01)$, and V4 $\left(F_{(1,11)}=11.22, p<0.01\right)$, demonstrating that the reward schedule biased toward the different-object location completely reversed the direction of the traditional object-based effect. It should be noted that the response pattern in V1 was similar to that observed in visual areas V2-V4 (i.e., numerical trend), even though it failed to reach statistical significance. This suggests that activity in V1 is not qualitatively different from that in later visual areas, but the effect size was not large enough because of relatively small receptive field size of $\mathrm{V} 1$ cells and insufficient statistical power.

The additivity of the modulatory effects of reward and objectbased attentional allocation was tested using the same procedure as in the behavioral analysis, confirming that the magnitude of modulation effect was not significantly different between SOand DO-rewarded schedules in all visual areas. These results demonstrate that the target-evoked activity within the sensory cortex is exclusively predicted by reward and not by reward and object interaction, providing strong evidence that object-based selection is a default setting and fails to exert its influence in the presence of an alternative reward-based signal.

\section{Behavioral and neural correlation analysis}

The relationship between behavioral effects and the corresponding attentional modulation of early sensory regions was assessed by examining whether the greater individual magnitude of reward modulation in the behavioral response resulted in a greater neural response. To this end, an index was derived reflecting the magnitude of reward-based modulation for each target type, quantifying individual differences in response to a certain target depending on the reward context. To compute the index, for each reward schedule separately, the response for each target type was normalized by dividing by the sum of all the responses (valid $+\mathrm{SO}$
+ DO). Then, the difference between responses to a certain target type across SO- and DO-rewarded schedules was calculated, subtracting the response to a particular target type when it was lowrewarded from when it was high-rewarded [response for SO targets (SO-rewarded - DO-rewarded), response for DO targets (DO-rewarded - SO-rewarded)]. We termed this index a Modulation Across Reward Schedules (MARS) and calculated each individual's MARS score for each target type separately for behavioral and neural data. IE data were used to calculate the behavioral MARS, and the averaged target-related activity across visual areas V2, V3, and V4 was used to calculate the corresponding neural MARS (V1 was excluded from the analysis because of lack of significance in reward schedule by target type interaction).

To examine the direct relationship between behavioral effects and the corresponding modulations within the early visual areas, behavioral MARS indices were correlated with neural MARS indices. Because IEs decreased whereas the neural response increased when a target was highly rewarded, behavioral and neural MARS indices were expected to have a negative relationship. Confirmatively, significant negative correlation between the behavioral and neural MARS indices for SO targets $(r=-0.73, p<0.01)$ and also for DO targets $(r=-0.60, p<0.05)$ was observed (Fig. 3), indicating that, as the extent of reward-based behavioral benefit increased, the extent of reward-based neural benefit in visual areas increased as well. The observed direct relationship provides strong evidence that reward modulates attentional allocation, thereby increasing processing efficiency for rewarded stimuli.

\section{Cue-related activity}

To examine whether the observed pattern of reward modulation effects is specific to target processing (previous section) or reflects preparatory effects of selective attention, cue-related activity was probed by extracting BOLD response at the time of the cue onset from POIs. Peak response to cue was calculated and submitted to ANOVA, revealing significant main effect of target type $\left(F_{(2,22)}=\right.$ $14.96, p<0.01)$, visual area $\times$ target type interaction $\left(F_{(6,66)}=\right.$ $3.33, p<0.01)$, and reward schedule $\times$ target type interaction $\left(F_{(4,44)}=3.43, p<0.02\right)$. No other effects reached significance $(F<1)$. Because the three-way interaction between visual area, reward schedule, and target type was not significant, data for further analysis were collapsed across areas V1-V4 (Fig. 4).

Planned comparisons revealed greater responses for valid targets than for invalid targets in random reward schedule $\left(F_{(1,11)}=\right.$ 23.06, $p<0.01)$, in SO-rewarded schedule $\left(F_{(1,11)}=7.59, p<\right.$ $0.02)$, and in DO-rewarded schedule $\left(F_{(1,11)}=13.71, p<0.01\right)$, demonstrating significant space-based effects, suggesting that it is in fact mandatory. These results differ from those observed in behavioral performance or in the target-related activity, in which a space-based effect was significant only in random reward schedule, and not in SO- and DO-rewarded schedules. ANOVA revealed a significant timing (cue-related, target-related) by reward schedule (random, SO-rewarded, DO-rewarded) interaction $\left(F_{(2,22)}=3.55, p<0.05\right)$ for space-based effects, supporting that the patterns of space-based effect were different between cue- and target-related activities. This apparent discrepancy reveals an important relationship between automatic space- and reward-based orienting; a space-based effect occurs mandatorily after the cue onset, but it is overridden by the presence of a reward-based strategy (SO- and DO-rewarded) over the time interval between the cue and the target, lingering only when reward is noninformative (random reward). This result strongly suggests that spatial orienting is mandatory and is initially (onset of the cue) robust to influences from reward, consistent with previous studies demonstrating automatic 
deployment of attention to cued location (Posner, 1980; Yantis and Jonides, 1990; Theeuwes, 1991), but is modulated by reward with sufficient time, as evidenced by the results in target-related activity.

Object-based effects, however, were absent even after the cue onset, as evidenced by the absence of a significant difference between responses for SO and DO targets in random reward schedule. Additionally, in the SO-rewarded schedule, the response for $\mathrm{SO}$ targets was greater than that for DO targets $\left(F_{(1,11)}=5.02, p<0.05\right)$, consistent with the direction of the traditional object-based effect. In the DOrewarded schedule, the response was greater for DO targets than for SO targets $\left(F_{(1,11)}=4.62, p<0.06\right)$, indicating a reversal of the direction of object-based effect. These results reveal an important difference between the interaction of reward and space- and objectbased attention, with space-based attention being modulated by reward with time, whereas object-based attention being abandoned immediately in lieu of reward.

The additivity of the effects of reward and object-based attentional allocation was tested, confirming that the magnitude of modulation effect was not significantly different between SO- and DOrewarded schedules. These results are consistent with the behavioral pattern as well as with the pattern observed in the target evoked response, strongly suggesting that object-based selection is a default setting and fails to exert its influence in the presence of an alternative reward-based signal. Also, the tight coupling between cue-related and target-related neural activity suggests that the reward-based modulation of object-based effects emerges immediately after the cue and persists until target identification.

\section{Multivoxel correlation analysis}

To probe whether the reward-based modulation pattern observed in the univariate analysis is also supported at a more sensitive and fine-grained voxel level, a multivoxel correlation analysis was performed following the method of Haxby et al. (2001), examining whether the spatial pattern of fMRI response at the voxel level differentiates between target types in different reward schedules (Fig. 5a). In all three reward schedules, the correlation coefficient between even and odd valid targets was the greatest, reflecting the attentional benefit for valid targets resulting from the highest target probability $(60 \%)$. Also, correlations were higher between the same types of targets (downward diagonals of the correlation matrices in Fig. $5 a$ ) than between different types of targets, indicating that the voxelwise pattern of activation in early visual areas successfully differentiated between target types. This result is remarkable considering that the physical stimulus (color patch) shown for each target type was exactly the same, which suggests that the spatial pattern of visual response contains information regarding attentional modulation.

Next, we examined whether signal integrity of each target type is also exclusively determined by reward schedules, by comparing the correlation coefficients of spatial pattern of response for each target type (Fig. 5b). A repeated-measures ANOVA revealed a significant main effect of target type $\left(F_{(2,22)}=18.64, p<0.01\right)$ and a significant reward schedule $X$ target type interaction $\left(F_{(4,44)}=4.93, p<0.01\right)$. No other main effects or interactions reached significance. Planned comparisons were further conducted for each reward schedule as follows: Random (Fig. 5b, left): the correlation coefficients were greater for valid targets than for SO targets $\left(F_{(1,11)}=18.38, p<0.01\right)$ or for DO targets

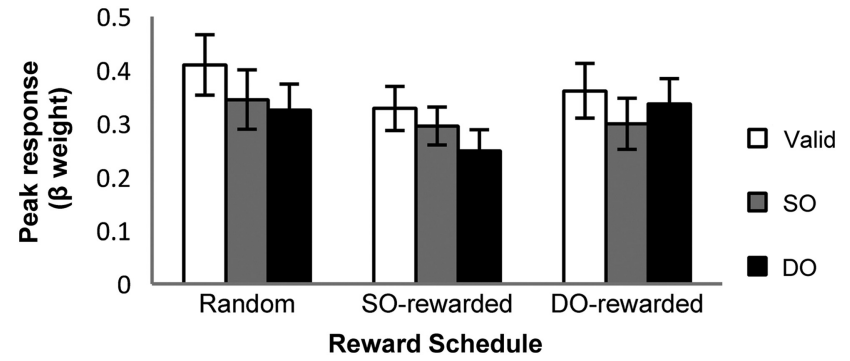

Figure 4. Cue-related peak responses for each target type (valid, SO, DO) in each reward schedule (Random, SO-rewarded, D0-rewarded) collapsed across visual areas.

$\left(F_{(1,11)}=17.46, p<0.01\right)$. Importantly, correlation coefficients for SO and DO targets were not significantly different from one another, indicating the absence of an object-based effect. SOrewarded (Fig. $5 b$, middle): the correlation coefficient for valid targets was greater than that for DO targets $\left(F_{(1,11)}=22.20, p<\right.$ $0.01)$. Also, the correlation coefficient for $\mathrm{SO}$ targets was greater than that for DO targets $\left(F_{(1,11)}=9.85, p<0.01\right)$, consistent with the direction of the traditional object-based effect. DO-rewarded (Fig. $5 b$, right): the correlation coefficient for valid targets was greater than that for SO targets $\left(F_{(1,11)}=12.39, p<0.01\right)$. Importantly, the correlation coefficient for DO targets was greater than that for SO targets $\left(F_{(1,11)}=11.74, p<0.01\right)$, indicating a complete reversal of the direction of object-based effect due to the reward schedule biased toward DO targets. These results are consistent with the pattern obtained in the univariate analysis, providing greater confidence and further support for the conclusions that space-based selection occurs mandatorily, whereas objectbased selection is abandoned in lieu of reward, and the rewardbased effect is accomplished in a systematic way that is consistent across voxels in any given ROI.

\section{Whole-brain analysis}

To examine representations of reward and attention outside of spatially organized visual areas, a whole-brain analysis, time locked to target onset, was conducted defining regions that exhibited greater activation for high-reward targets than for lowreward targets. The resulted regions (Table 1) include areas typically associated with attention and reward, including right frontal eye field (rFEF), right medial frontal gyrus (rMFG), right superior frontal gyrus (rSFG), right insula (rInsula), right anterior cingulate (rACC), left precuneus, and left inferior parietal lobule (IIPL) (Schultz et al., 1998; Corbetta and Shulman, 2002; Hikosaka, 2007; Gottlieb and Balan, 2010).

To investigate whether the magnitude of modulation in reward regions is related to that observed within the early visual areas, we extracted target-evoked neural activity from each ROI identified in the whole-brain analysis, calculated the MARS index for each target type, and correlated it with the MARS index in the 
a

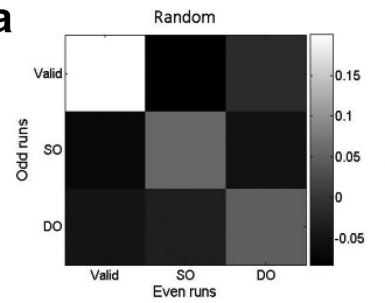

b

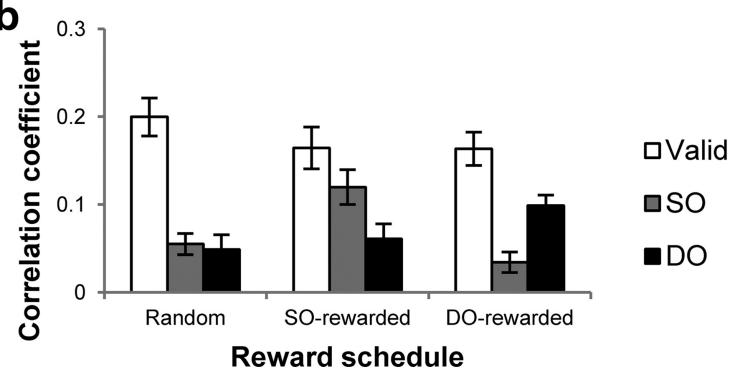

Figure 5. a, Correlation matrix obtained from the multivoxel correlation analysis. It illustrates correlation coefficients between every possible pair of target types, comparing each target type in the odd runs with each target type in the even runs. $\boldsymbol{b}$, Correlation coefficients of each target type between even and odd runs in three reward schedules.

early visual areas. Four of the eight identified ROIs showed a direct relationship with activity in the early visual areas: IIPL, rACC, rInsula, and rSFG. Positive correlations between the MARS indices for SO targets were observed for IIPL $(r=0.65, p<$ $0.05)$, rACC $(r=0.63, p<0.05)$, rInsula $(r=0.69, p<0.05)$, and rSFG $(r=0.65, p<0.05)$ (Fig. 6, top). Positive correlations between the MARS indices for DO targets were also observed for IIPL $(r=0.67, p<0.05)$, rInsula $(r=0.62, p<0.05)$, and $\mathrm{rSFG}$ $(r=0.64, p<0.05)$ (Fig. 6 , bottom). The direct relationship strongly supports that identified regions influence selection by issuing control signals that modulate activity in early sensory areas. Although correlation findings do not imply causality, these results are consistent with the theoretical framework of attentional selection that frontoparietal areas issue transient signals that initiate shifts of attention (Yantis et al., 2002; Shomstein and Behrmann, 2006) and are compatible with the many recent findings showing reward-based modulation in early regions of visual cortex (Shuler and Bear, 2006; Serences, 2008).

Among the regions identified in the whole-brain analysis, $\mathrm{rFEF}$ and IIPL have been repeatedly reported as providing attentional biasing signal (Corbetta and Shulman, 2002). We examined whether the attentional biasing signal in these regions is also exclusively driven by reward or by additive effects of reward and object-based attention (Fig. 7). The results demonstrated that activity in the regions was modulated exclusively by reward. This finding provides further evidence and strongly corroborates the conclusion that, in case of object-based attention, reward schedule exclusively modulates the biasing signal that directs attentional selection.

\section{Discussion}

Results of a series of fMRI experiments reported here provide strong evidence that reward-based orienting serves as an informative signal that constrains or even overrides attentional selection. Importantly, we show that the effect of reward on selection varies depending on the type of attentional guidance: space-based selection is modulated by reward; whereas object-based selection is abandoned in favor of a reward-based contingency.

Two major conclusions are drawn from the current findings. First, space-based attentional guidance occurs mandatorily and interacts with reward, supported by the observation that space- based effects occurred after the cue but disappeared with time. This result dovetails with decades of attentional selection research demonstrating automaticity of space-based attention (Posner, 1980; Yantis and Jonides, 1990; Theeuwes, 1991), complemented with a novel finding that space-based effect is eventually modulated by influences from reward with sufficient time. This result indicates that even highly automatic attentional guidance succumbs to reward-based modulation.

The second major conclusion is that object-based attentional guidance, rather than interacting with reward, is completely abandoned in favor of the reward contingency. Reward-based modulation emerged immediately after a cue and persisted until target identification, suggesting that perceptual efficiency was determined exclusively by reward. Contrary to suggestions of object-based automaticity (Chen and Cave, 2008; Yeari and Goldsmith, 2010), reward schedule exclusively modulated neural activity as well as behavioral performance, providing strong evidence that object-based selection is a default setting that occurs under idealized conditions (i.e., all else being equal) (Shomstein and Johnson, 2013).

It could be argued that the pattern of results observed in this experiment might be consistent with another set of simple or complex interactions of reward, probability (spatial orienting), or object information (object representation). To rule out this interpretation, we generated a set of clear predictions based on each type of strategy and compared it against the observed findings. In random reward schedule, reward alone predicts an equal amount of benefit for valid, SO, and DO targets because reward would be distributed randomly and equally (3.5 points on average) across all possible target locations. Probability alone predicts benefit for valid targets (0.6) followed by equal benefit for SO $(0.2)$ and DO (0.2) targets. Such patterns will be consistent across all three reward schedules. Object-based contribution predicts an additional advantage for SO targets over DO targets. Reward $\times$ probability interaction would favor valid $(3.5 \times 0.6=2.1)$ targets the most, followed by equal benefit for SO $(3.5 \times 0.2=0.7)$ and DO $(3.5 \times 0.2=0.7)$ targets. Reward $\times$ probability $\times$ object interaction would predict similar results to reward $\times$ probability with an added benefit of SO over DO targets. In SO-rewarded schedule, reward alone predicts the maximal benefit for SO (6 points) targets, followed by valid (3.5 points on average) targets, and the least benefit for DO (1 point) targets. Probability is the same in all three reward schedules. Object-based contribution predicts an additional advantage for SO targets over DO targets. Reward $\times$ probability interaction would result in the greatest benefit for valid $(3.5 \times 0.6=2.1)$ targets, followed by SO $(6 \times$ $0.2=1.2)$ targets, and the least benefit for DO $(1 \times 0.2=0.2)$ targets. Reward $\times$ probability $\times$ object interaction would predict an added benefit of SO over DO targets, leading to an overexaggerated object-based effect. In DO-rewarded schedule, reward alone predicts the maximal benefit for DO targets (6 points), followed by valid ( 3.5 points on average) targets, and the least benefit for SO (1 point) targets. Probability is the same in all three reward schedules. Object-based contribution predicts an additional advantage for SO targets over DO targets. Reward $\times$ prob- 

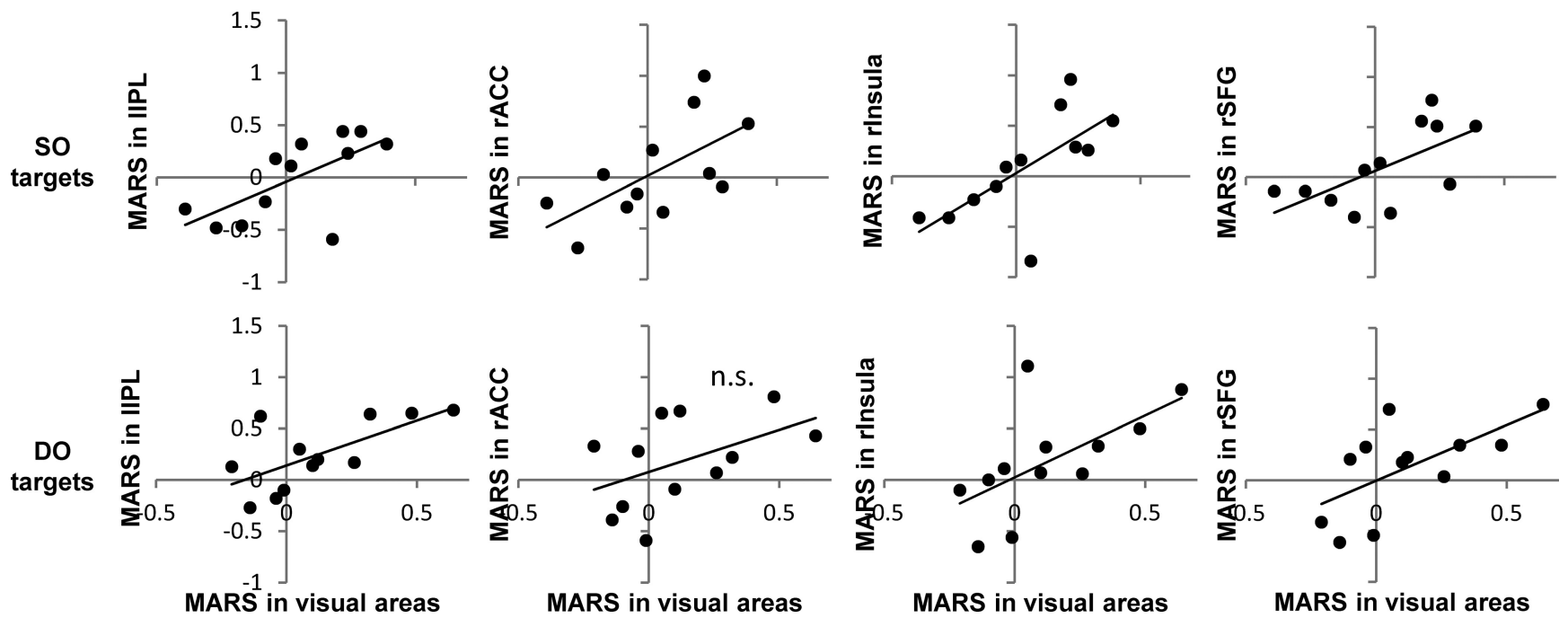

Figure 6. Scatterplots illustrating the relationship between the magnitude of reward modulation in the reward-related regions and in the early visual areas. Top, The relationship between MARS indices for $\mathrm{SO}$ targets. Bottom, The relationship between MARS indices for D0 targets. The first, second, third, and fourth columns represent the relationship between MARS indices in early visual areas and in IIPL, rACC, rlnsula, and rSFG, respectively. Significant positive correlations were observed in all regions, except MARS indices for D0 targets in rACC (indicated by n.S.).

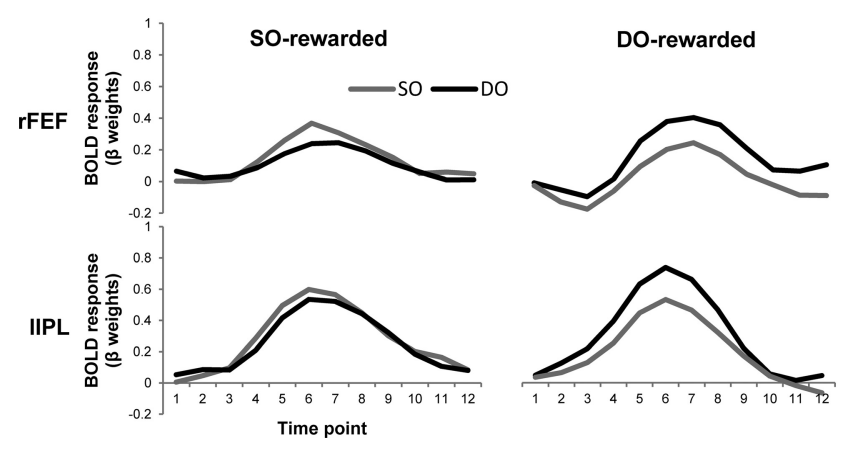

Figure 7. Target-related BOLD time courses for each target type (SO, DO) in each reward schedule (SO-rewarded, D0-rewarded) in rFEF (top) and in IIPL (bottom).

ability interaction would result in the greatest benefit for valid $(3.5 \times 0.6=2.1)$ targets, followed by DO $(6 \times 0.2=1.2)$ targets, and the least benefit for SO $(1 \times 0.2=0.2)$ targets. Reward $\times$ probability $\times$ object interaction would predict similar results to reward $\times$ probability with a caveat that DO over SO benefit will either decrease or be eliminated.

The pattern of the cue-related data was most consistent with the reward $\times$ probability hypothesis, which persisted until target identification only when reward was noninformative (random reward). When reward was informative (SO- and DO-rewarded), on the other hand, the weighting for reward increased, whereas the weighting for probability decreased over the time interval between the cue and the target, aligning the target-related behavioral and neural data more closely with the predictions of the reward-only hypothesis. These results strongly support that participants used an optimal strategy that maximized the harvested reward in each of the three reward schedules, integrating the effects of reward and other attentional guidance. Importantly, multiple sources of information are integrated dynamically. For instance, space-based effects observed in SO- and DO-rewarded schedules after the cue onset disappeared after the target onset, indicating that the weighting for probability information decreased over the cue-to-target interval. Also, objectbased contribution observed in the behavioral experiment (without reward) disappeared once reward schedules were introduced, indicating that the weighting for object information dropped to zero when more reliable reward information was added to the equation. The dynamic nature of the weighting process argues against a possible speculation that effects of reward can be likened to any other endogenous attentional orienting. For instance, the weight of reward might have been much larger than that of probability or object information in the context of our experiment. Thus, different pattern of results would have been observed if only a spatial cue (indicating multiple locations) was used compared with when both spatial cue and reward cue were used. Empirical data collected here suggest that information from multiple sources is integrated in an optimal way, such that different weighting is given to each source based on the relevance and significance of that information in the current situation (Navalpakkam et al., 2010; Gottlieb, 2012).

Despite a growing body of literature on the relationship between modulating effects of reward and attention, most studies have focused on either behavioral or neural mechanisms. By developing an experimental paradigm that allowed examining the behavioral and neural effects of reward and attention simultaneously, for the first time, we provide comprehensive behavioral and neural evidence that is consistent across multiple levels of analysis. First, the nonadditivity of the modulating effects of reward and default attentional guidance was observed consistently in the behavioral and neural measures. Moreover, a positive correlation between the magnitude of reward-based modulation in behavioral and neural measures was observed, demonstrating the tight coupling between behavioral and neural benefit for rewarded stimuli. Second, the same pattern of results was observed at multiple levels of analysis, such that reward schedule exclusively modulated the neural activity across early visual areas, both in univariate analysis and in multivoxel correlation analysis, corroborating that reward-based selection provides an informative signal that takes over attentional allocation in a systematic way that is consistent across voxels. Finally, reward exclusively modulated neural activity in ROIs that provide attentional biasing signal, and the magnitude of modulation in those regions was positively related to that observed within the early visual areas. Taken together, our results present comprehensive and converging evidence that reward provides an informative signal that constrains or even overrides automatic attentional allocation, such 
that some forms of attentional guidance are completely abandoned in lieu of a reward-based strategy.

The striking result that reward structure supersedes objectbased attentional allocation leads to a provocative suggestion that reward-based selection should not be viewed separately from attention; rather, it should be conceptualized as one of the units of attention. These results are particularly timely given the growing debate on whether separate, or single integrated, priority maps are constructed based on reward and attention (Platt and Glimcher, 1999; Gottlieb and Balan, 2010; Gottlieb, 2012). Most previous work, however, investigated the modulating effects of reward and attention independently (Müller and Kleinschmidt, 2003; Dorris and Glimcher, 2004; Della Libera and Chelazzi, 2006; Goldberg et al., 2006), yielding inconsistent conclusions. Studies using decision-making paradigms, for instance, maintained that lateral intraparietal area contains a salience map that incorporates reward information exclusively, independent of behavioral relevance (Platt and Glimcher, 1999; Dorris and Glimcher, 2004). This line of research assumes that visual attention is conceptually separable from reward-related process, and hypothesizes independent priority maps constructed either by reward or attention alone. On the other hand, several studies proposed that reward can be conceptualized as one of the units of attention, by demonstrating that reward manipulation modulated activity in brain regions related to top-down control of attention (Small et al., 2005; Kiss et al., 2009). This line of research hypothesizes existence of a single integrated priority map that incorporates both reward and attentional information (Della Libera and Chelazzi, 2006; Peck et al., 2009; Gottlieb and Balan, 2010). In the current study, activity in regions providing attentional biasing signal was exclusively driven by reward, providing further support that the effects of reward and other attetional guidance are not independent. Systematic results of the current study showing that default attentional selection is completely abandoned in lieu of reward-based strategy, instead of manifesting in an additive manner, provide supporting evidence for a single priority map that unifies information from all the contributing signals. Reward, we argue, is one of the contributing signals for attentional selection, similar to other units of attention.

\section{References}

Behrmann M, Zemel RS, Mozer MC (1998) Object-based attention and occlusion: Evidence from normal participants and a computational model. J Exp Psychol Hum Percept Perform 24:1011-1036. CrossRef Medline

Chen Z, Cave KR (2008) Object-based attention with endogenous cuing and positional certainty. Percept Psychophys 70:1435-1443. CrossRef Medline

Corbetta M, Shulman GL (2002) Control of goal-directed and stimulusdriven attention in the brain. Nat Rev Neurosci 3:201-215. CrossRef Medline

Della Libera C, Chelazzi L (2006) Visual selective attention and the effects of monetary rewards. Psychol Sci 17:222-227. CrossRef Medline

Dorris MC, Glimcher PW (2004) Activity in posterior parietal cortex is correlated with the relative subjective desirability of action. Neuron 44:365378. CrossRef Medline

Egeth HE, Yantis S (1997) Visual attention: control, representation, and time course. Annu Rev Psychol 48:269-297. CrossRef Medline

Egly R, Driver J, Rafal RD (1994) Shifting visual attention between objects and locations: evidence from normal and parietal lesion subjects. J Exp Psychol Gen 123:161-177. CrossRef Medline

Goebel R, Esposito F, Formisano E (2006) Analysis of functional image analysis contest (FIAC) data with brainvoyager QX: from single-subject to cortically aligned group general linear model analysis and self-organizing group independent component analysis. Hum Brain Mapp 27:392-401. CrossRef Medline

Goldberg ME, Bisley JW, Powell KD, Gottlieb J (2006) Saccades, salience and attention: the role of the lateral intraparietal area in visual behavior. Prog Brain Res 155:157-175. CrossRef Medline

Gottlieb J (2012) Attention, learning, and the value of information. Neuron 76:281-295. CrossRef Medline

Gottlieb J, Balan P (2010) Attention as a decision in information space. Trends Cogn Sci 14:240-248. CrossRef Medline

Haxby JV, Gobbini MI, Furey ML, Ishai A, Schouten JL, Pietrini P (2001) Distributed and overlapping representations of faces and objects in ventral temporal cortex. Science 293:2425-2430. CrossRef Medline

Hickey C, Chelazzi L, Theeuwes J (2010) Reward changes salience in human vision via the anterior cingulate. J Neurosci 30:11096-11103. CrossRef Medline

Hikosaka O (2007) Basal ganglia mechanisms of reward-oriented eye movement. Ann N Y Acad Sci 1104:229-249. CrossRef Medline

Kastner S, Ungerleider LG (2000) Mechanisms of visual attention in the human cortex. Annu Rev Neurosci 23:315-341. CrossRef Medline

Kiss M, Driver J, Eimer M (2009) Reward priority of visual target singletons modulates event-related potential signatures of attentional selection. Psychol Sci 20:245-251. CrossRef Medline

Kristjánsson A, Sigurjónsdóttir O, Driver J (2010) Fortune and reversals of fortune in visual search: reward contingencies for pop-out targets affect search efficiency and target repetition effects. Atten Percept Psychophys 72:1229-1236. CrossRef Medline

Moore CM, Yantis S, Vaughan B (1998) Object-based visual selection: evidence from perceptual completion. Psychol Sci 9:104-110. CrossRef

Müller NG, Kleinschmidt A (2003) Dynamic interaction of object- and spacebased attention in retinotopic visual areas. J Neurosci 23:9812-9816. Medline

Navalpakkam V, Koch C, Rangel A, Perona P (2010) Optimal reward harvesting in complex perceptual environments. Proc Natl Acad Sci U S A 107:5232-5237. CrossRef Medline

Peck CJ, Jangraw DC, Suzuki M, Efem R, Gottlieb J (2009) Reward modulates attention independently of action value in posterior parietal cortex. J Neurosci 29:11182-11191. CrossRef Medline

Platt ML, Glimcher PW (1999) Neural correlates of decision variables in parietal cortex. Nature 400:233-238. CrossRef Medline

Posner MI (1980) Orienting of attention. Q J Exp Psychol 32:3-25. CrossRef Medline

Ress D, Backus BT, Heeger DJ (2000) Activity in primary visual cortex predicts performance in a visual detection task. Nat Neurosci 3:940-945. CrossRef Medline

Schultz W, Tremblay L, Hollerman JR (1998) Reward prediction in primate basal ganglia and frontal cortex. Neuropharmacology 37:421-429. CrossRef Medline

Serences JT (2008) Value-based modulations in human visual cortex. Neuron 60:1169-1181. CrossRef Medline

Serences JT, Shomstein S, Leber AB, Golay X, Egeth HE, Yantis S (2005) Coordination of voluntary and stimulus-driven attentional control in human cortex. Psychol Sci 16:114-122. CrossRef Medline

Sereno MI, Dale AM, Reppas JB, Kwong KK, Belliveau JW, Brady TJ, Rosen BR, Tootell RB (1995) Borders of multiple visual areas in humans revealed by functional magnetic resonance imaging. Science 268:889-893. CrossRef Medline

Shomstein S, Behrmann M (2006) Cortical systems mediating visual attention to both objects and spatial locations. Proc Natl Acad Sci U S A 103: 11387-11392. CrossRef Medline

Shomstein S, Johnson J (2013) Shaping attention with reward: effects of reward on space- and object-based selection. Psychol Sci, in press.

Shomstein S, Yantis S (2004) Configural and contextual prioritization in object-based attention. Psychon Bull Rev 11:247-253. CrossRef Medline

Shuler MG, Bear MF (2006) Reward timing in the primary visual cortex. Science 311:1606-1609. CrossRef Medline

Small DM, Gitelman D, Simmons K, Bloise SM, Parrish T, Mesulam MM (2005) Monetary incentives enhance processing in brain regions mediating top-down control of attention. Cereb Cortex 15:1855-1865. CrossRef Medline

Talairach J, Tournoux P (1988) Co-planar stereotaxic atlas of the human brain. New York: Thieme.

Theeuwes J (1991) Exogenous and endogenous control of attention: the effect of visual onsets and offsets. Percept Psychophys 49:83-90. CrossRef Medline

Townsend JT, Ashby FG (1983) Stochastic modelling of elementary psychological processes. New York: Cambridge UP. 
Yantis S, Jonides J (1990) Abrupt visual onsets and selective attention: voluntary versus automatic allocation. J Exp Psychol Hum Percept Perform 16:121-134. CrossRef Medline

Yantis S, Schwarzbach J, Serences JT, Carlson RL, Steinmetz MA, Pekar JJ, Courtney SM (2002) Transient neural activity in human parietal cortex during spatial attention shifts. Nat Neurosci 5:995-1002. CrossRef Medline

Yeari M, Goldsmith M (2010) Is object-based attention mandatory? Strategic control over mode of attention. J Exp Psychol Hum Percept Perform 36:565-579. CrossRef Medline 